Mon. Not. R. Astron. Soc. 000, 1 17(2015) Printed 27 May $2022 \quad$ (MN LATEX style file v2.2)

\title{
The Shape of Dark Matter Haloes III. Kinematics and Structure of the HI disc
}

\author{
S. P. C. Peters ${ }^{1}$, P. C. van der Kruit ${ }^{1 \star}$, R. J. Allen ${ }^{2}$ and K. C. Freeman ${ }^{3}$ \\ ${ }^{1}$ Kapteyn Astronomical Institute, University of Groningen, P.O.Box 800, 9700AV Groningen, the Netherlands \\ ${ }^{2}$ Space Telescope Science Institute, 3700 San Martin Drive, Baltimore, MD 21218, USA \\ ${ }^{3}$ Research School of Astronomy and Astrophysics The Australian National University, Cotter Road Weston Creek, ACT 2611, \\ Australia
}

Accepted 2015 month xx. Received 2015 Month xx; in original form 2015 Month xx

\begin{abstract}
We present a new strategy for fitting the structure and kinematics of the HI in edgeon galaxies using a fit to the terminal-velocity channel maps of a HI data cube. The strategy can deal with self-absorbing HI gas and the presence of warps. The method is first tested on a series of models. We demonstrate that fitting optically thin models to real galaxies will lead to an overestimation of the thickness and velocity dispersion, and to a serious underestimation of the HI face-on column densities. We subsequently fit both self-absorption and optically thin models to the HI data of six edge-on galaxies. In three of these we have also measured the velocity dispersion. On average $27 \pm 6 \%$ of the total HI mass of edge-on galaxies is hidden by self-absorption. This implies that the HI mass, thickness and velocity dispersion of galaxies is typically underestimated in the literature.
\end{abstract}

Key words: galaxies: haloes, galaxies: kinematics and dynamics, galaxies: photometry, galaxies: spiral, galaxies: structure

\section{INTRODUCTION}

In Paper I in this series, we have presented the HI observations for eight edge-on late-type galaxies. One of our main conclusions was that the HI showed clear signs of self-absorption, which created the risk of a rather drastic underestimation of the baryonic content of the galaxy. We developed a new HI modelling and fitting system called Galactus in Paper II to address this problem. We showed that the HI self-absorption indeed has a drastic impact on the observed maximum surface brightness temperature. Selfabsorption needs to be taken into account when modelling the HI in edge-on galaxies.

The main goal of our project throughout this series of papers is to measure the hydrostatics at the central plane of edge-on galaxies. To get to this, we will need to derive the rotation curve $v_{\text {rot }}(R)$, face-on surface density $A_{\mathrm{HI}}(R)$, thickness of the HI layer $z_{0}(R)$ and its velocity dispersion $\sigma(R)$ accurately. Secondary parameters, such as the exact kinematic position in RA, DEC and the systemic velocity $v_{\text {sys }}$, will also need to be derived. While warps are of scientific interest, they represent a disturbance of the central plane of the galaxy. The physics behind warps is not well

\footnotetext{
* For more information, please contact P.C. van der Kruit at email vdkruit@astro.rug.nl.
}

understood (van der Kruit \& Freeman 2011). Using the hydrostatics from the warped region would be a dangerous and ultimately futile exercise. We therefore refrain from modelling the warps. The channel maps of the observations, with the model superimposed on it, will be used to estimate the position at which the warp sets in. Beyond this position, we will not calculate the hydrostatics.

Fitting the HI structure and kinematics of edge-on galaxies by modelling the position-velocity (XV) diagram has a long-standing record of accomplishment. Various methods have been devised, initially only to derive the rotation curve and the radial distribution of HI surface brightness, and in some cases also the flaring (the increasing thickness as a function of galactocentric radius) of the HI layer (e.g. Sancisi \& Allen 1979 van der Kruit 1981, García-Ruiz et al. 2002 || Takamiya \& Sofue 2002||Uson \& Matthews 2003. Kregel \& van der Kruit 2004 Kregel et al.|2004, and references therein). More recently, Olling (1996a b) and O'Brien et al. (2010 b a c c) have measured the HI velocity dispersion. The paper by O'Brien et al. (2010b) provides a detailed description of the various methods and a discussion on the relative merits and pitfalls.

In this paper, we develop a new approach to modelling the HI properties of an edge-on galaxy, which consists of fitting channel maps near the terminal velocity of the galaxy. In Section 2, we explain this fitting strategy in more detail. 
Section 3 then applies this strategy on a series of test models and demonstrates the validity of the method. The kinematics and structure of six edge-on galaxies is subsequently presented in Section 4

\section{FITTING STRATEGY FOR EDGE-ON GALAXIES}

In Paper II, we demonstrated that the face-on galaxy NGC 2403 has at least 10\% more HI mass than an optically thin model would have measured. Rotating NGC 2403 to an edge-on geometry and assuming a spin temperature of $100 \mathrm{~K}$, we found that $22 \%$ of the total HI mass would have been hidden by self-absorption. A lower median spin temperature of $80 \mathrm{~K}$ increases this fraction to $30 \%$ (see Section 7 of Paper II). Any attempt to model an edge-on galaxy as optically thin will thus suffer from a drastic underestimation of total HI mass.

While this is troubling enough by itself, other questions regarding the effect of the self-absorption can also be raised:

- What is the effect of self-absorption on the rotationcurve estimate?

- Is the thickness of the disc still measured properly?

- What is the impact on the measured velocity dispersion?

A common practice for measuring the kinematics of edge-on galaxies is through the outer envelope of the (integrated) XV diagram, either adopting the peak flux as the rotation at the line of nodes, or by fitting a halfGaussian to the terminal velocities (e.g. Sancisi \& Allen 1979, Olling 1996a; Kregel \& van der Kruit 2004, O'Brien et al. $2010 \mathrm{~b}$ a and references therein). Both the velocity dispersion and thickness of the disc can change the mid-plane surface brightness in a particular channel. This creates a degeneracy between the two parameters. As such, there is no practical way to correct for the self-absorption using only the mid-plane or integrated XV-diagrams of a galaxy. We therefore opt for a different strategy and model the channelmaps of the HI data cube directly. An advantage to this strategy is that we can fit all four main parameters (i.e. the rotation curve, face-on surface density, velocity dispersion and the thickness) simultaneously and self-consistently. After much trial-and-error, we have devised the following three-pass strategy for fitting edge-on galaxies, using the Galactus fitting code developed in Paper II.

In the first pass, we fix the velocity dispersion at a constant value of $10 \mathrm{~km} / \mathrm{s}$ and fit the rotation curve, face-on surface density and thickness of the disc. The central position $\left(x, y, v_{\text {sys }}\right)$ along with the position angle $P A$ are also fitted during this pass. Tests have shown that measuring the exact inclination is difficult, so we fix the inclination at $90^{\circ}$. We fit the entire HI data cube. The galaxy has already been projected such that the major axis aligns with the horizontal axis as already discussed in Paper I.

The results are subsequently inspected and corrections made where necessary. We next fix the position and position angle. The galaxy is split in two halves, which will be fit separately. Based on the results from the initial fit, we create a mask, such that the terminal velocity is still visible at all radii, but the radii at lower rotational velocities
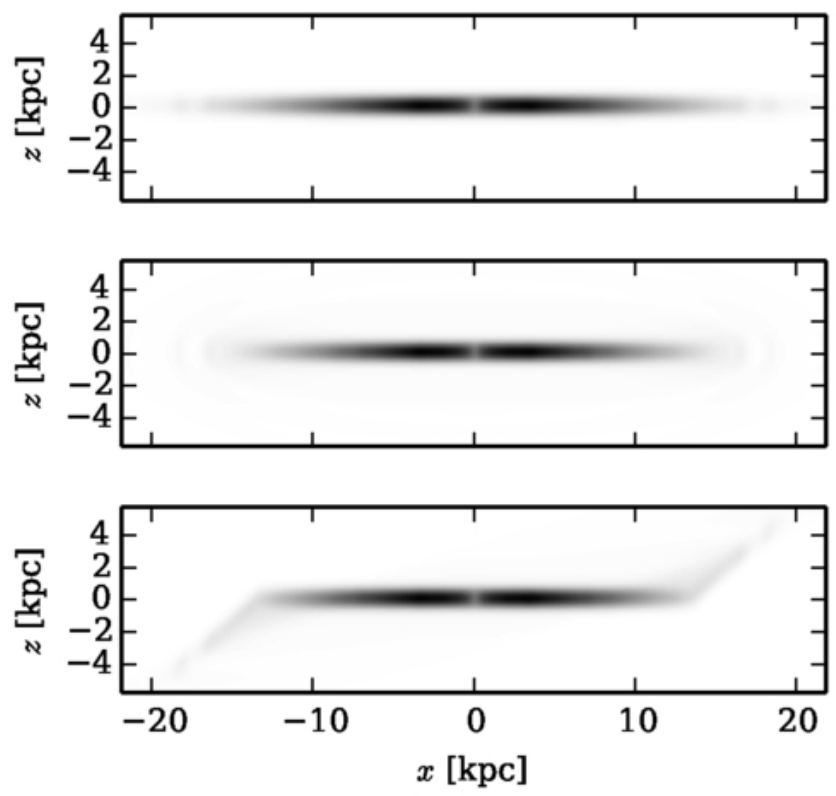

Figure 1. Moment-zero maps of a model without warp (top), face-on warp (middle) and a side-on warp (bottom).

are masked. The line-of-nodes velocity is expected near the terminal velocity. We do this because warps and asymmetries, together with the self-absorption, lead to degeneracies in the solution and thus can confuse the fitting algorithm. The masking is done by hand. Results from subsequent fits are inspected to ensure the line of nodes rotation is well beyond the masked positions. This is effectively creating an outer-envelope mask. Rather than just mask the XV diagram, we are now masking the HI data cube itself. After both sides have their HI data cubes masked, we fit the rotation curve, thickness, face-on surface density and velocity dispersion. The results are again inspected, corrected and refit where necessary.

To illustrate the need for an outer-envelope mask, we use Galactus to model various types of warps. We begin with the parameters of the model from Section 7 of Paper II. This model is based on the HI in NGC 2403, as determined by us using a fitting of the data taking self-absorption into account. The models will be simulated as perfectly edge-on. We generate three models: The first will have no warp, while the second will have a strong line-of-sight warp (the maximum deviation from the mid-plane occurs along the line of sight) and the third one a strong side-on warp (the maximum deviation from the mid-plane occurs perpendicular to the line of sight). The models are run in self-absorption mode at a spin temperature of $100 \mathrm{~K}$. Both warps begin at a radius of $13.6 \mathrm{kpc}$ from the centre of the galaxy. The offset of the warp above the plane the increases linearly with one $\mathrm{kpc}$ height for every one $\mathrm{kpc}$ radius. It peaks at a radius of $20.4 \mathrm{kpc}$ at a height of $6.8 \mathrm{kpc}$. In Figure 1, we demonstrate the zeroth-moment maps for the three models. The presence of a line-of-sight warp is very hard to detect from this image, as is typical for these types of warps (e.g. Gentile et al. 2003 and references therein). In contrast, the side-on warp is clearly visible.

Shown in Figure 2 are the height-integrated XV diagrams of the three models. While some variation is present 

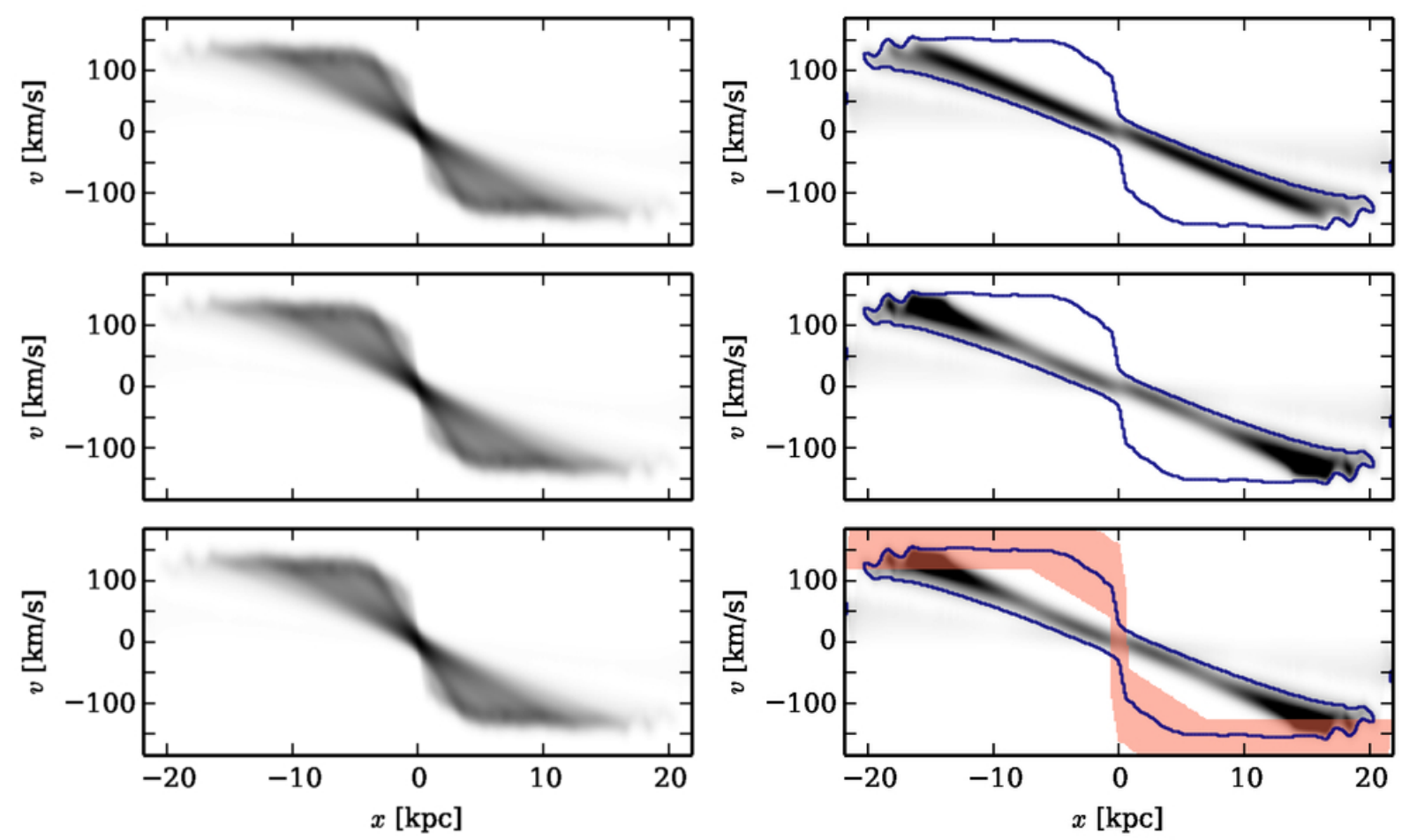

Figure 2. Left: Integrated XV-diagrams for a model without a warp (top), a line-of-sight warp (middle) and a side-on warp (bottom). Right: Integrated XV diagram of the difference HI data cube between the non-warped and face-on warp model (top), and the nonwarped and side-on warp model (middle). The blue contours show the outline of the full XV diagram. The bottom panel reproduces the middle panel, but has the envelope mask superimposed on top of it. Note that the fitting occurs on the channel maps rather than the XV-diagram.

between the three images, the differences are minor. To illustrate the effect of the warp, we have created two difference $\mathrm{XV}$-maps in Figure 2, Here we have subtracted the nonwarped HI data cube from both warped HI data cubes and integrated the absolute values of these differences along the minor axis. Both warps create a different signature in these difference maps. The line-of-sight warp creates a bar spread evenly over all lower velocity channels, while the side-on warp is far more pronounced at larger positions of $x$. Unsurprisingly, beyond $x>13.6 \mathrm{kpc}$ the warps affect the terminalvelocity channels as well and will thus affect the parameters extracted from this region. However, as demonstrated by the superimposed mask in the last panel, the outer envelope of the HI data cube at radii less than $13.6 \mathrm{kpc}$ is not affected by the warp.

Using an envelope mask of the HI data cube, we can thus measure the parameters of an edge-on galaxy inside the warped region, regardless of the presence of either a line-of-sight or side-on warp. Had we not masked the lower velocities, then the effect of the warps would have 'confused' the fitting algorithm.

In the final pass, the results are sampled with the Monte-Carlo Markov-Chain (MCMC) code EMCEE, which is used to sample the likelihood distribution in each parameter (see Section 2.8 of Paper II for more details). The results, auto-correlation and traces are inspected after a sufficient number of samples (i.e. 100.000+) have been drawn. When the MCMC has settled into a stable distribution, the final 10.000 samples are used to calculate the parameter distributions. We visualize these parameter distribution in sub- sequent figures based on the central $68.3,95.5$ and $99.7 \%$ fractions.

We fit each galaxy in two ways: using both an optically thin model and a self-absorption model with a spin temperature of $100 \mathrm{~K}$. As the physics of warps is not well understoo $^{1}$ we do not concern ourselves with warps. We fit the entire galaxy and determine by eye at what radius the warp starts to affect the flaring measurement. Beyond that radius, the data is considered unreliable.

\section{TESTING THE STRATEGY}

\subsection{High $\mathrm{S} / \mathrm{N}$ optically thin fit}

We first test our strategy on a series of models. We begin with an optically thin model, with a very low noise at $\sigma=0.1 \mathrm{~K}$. The model resembles UGC 7321, except that the linear scale is twice as small. The data is modelled at a distance of $10 \mathrm{Mpc}$, with pixels of 6.8 ", a channel width of $3.3 \mathrm{~km} / \mathrm{s}$ and a FWHM beam of 13.8 ". At only $48 \mathrm{~K}$, the maximum surface brightness in the model is still low, but the maximum signal to noise is very high at 480 . We present the results of the fit to this model in Figure 3 . As is clear from the image, the fit matches the initial near perfectly. Only at very large radii, where the HI surface density is lower than about $10^{20}$ atoms $/ \mathrm{cm}^{2}$, do we find a sudden increase in FWHM and decrease in $\sigma$ that are not present in 

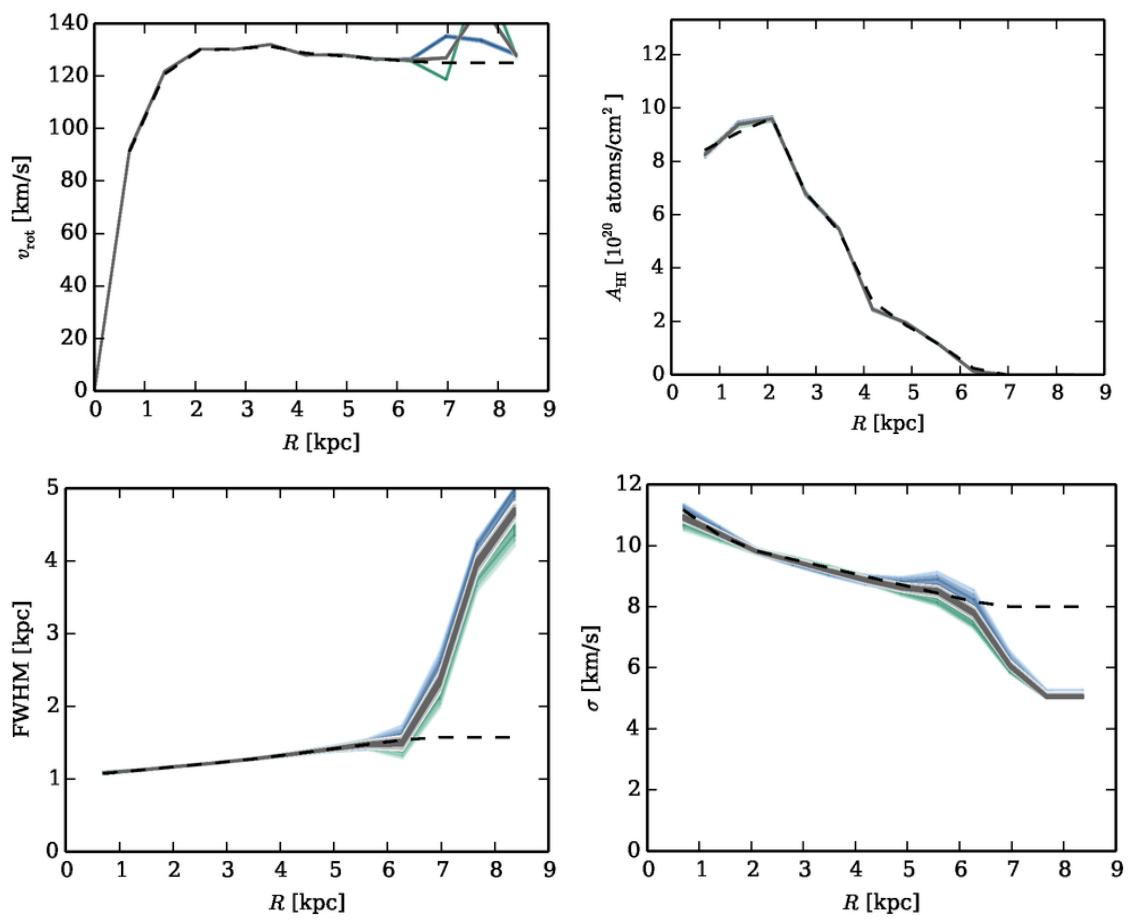

Figure 3. Results from a fit to both sides of an optically thin test model with low noize $(0.1 \mathrm{~K})$. Top-left panel shows the rotation curve $v_{\text {rot }}$, top-right panel the face-on surface density $A_{\mathrm{HI}}$. The lower-left panel shows the FWHM thickness of the disc. The velocity dispersion $\sigma$ is shown in the lower-right panel. Green colours denote the left side of the galaxy, blue colours show the right side. The grey band is the combined result. Dotted lines show the true parameters of the model.

the model. For such levels, where nearly no HI is present the fits become unreliable. We do find such behaviour in noisier models and in actual fits on our data below, usually also at levels below $10^{20}$ atoms $/ \mathrm{cm}^{2}$. Obviously such results should not and will not be used in any further modelling.

\subsection{Optically Thin Fits}

So how does the fitting deteriorate when the noise becomes higher? We double the size of the model from the previous test, such that the radial distance of each value of the parameters is twice as far out. The cell size is also doubled to 13.8". The model now has the same linear size as UGC 7321, on which it is based. This has the effect of raising the maximum surface brightness temperature to a more realistic value $\sim 90 \mathrm{~K}$. The models are run with noise levels of 1,3 and $5 \mathrm{~K}$. The maximum signal to noise then becomes 93, 30 and 20. The parameters found in the fits on either sides are smoothed. To create the combined parameters, the unsmoothed fits to either side are averaged together and the combined results smoothed. For the rotation curve, flaring and face-on surface density with a kernel of $[1 / 4,1 / 2,1 / 2]$ and the velocity dispersion with a kernel of $[1 / 3,1 / 3,1 / 3]$, as these parameters are found to be more sensitive to noise. For the combined result, both sides of the galaxy are averaged together from the unsmoothed fits, and only then is the combined data smoothed. We show the results for this fit in Figure 4 The rotation curve and the face-on surface density are recovered well in all cases. Measuring the thickness of the disc works reasonably well for the 1 and $3 \mathrm{~K}$ models - at least out to $10-12 \mathrm{kpc}-$, but the $5 \mathrm{~K}$ result starts to devi- ate more. The velocity dispersion is the hardest parameter to fit. Only for the $1 \mathrm{~K}$ model is the input model recovered sufficiently well out to $10 \mathrm{kpc}$, the $3 \mathrm{~K}$ result is marginal and the $5 \mathrm{~K}$ result is doubtful even at $8 \mathrm{kpc}$.

\subsection{Self-absorption Fits}

We perform a similar test using these models, but include self-absorption at a median spin temperature of $100 \mathrm{~K}$. The face-on surface density is increased to compensate for the self-absorption and now peaks around $1.5 \times 10^{20}$ atoms $/ \mathrm{cm}^{2}$. We show the results in Figure 5 . We find that the rotation curve is recovered well in all three models. Both the measurements of face-on surface density and those of the velocity dispersion become increasingly difficult at lower signal-tonoise ratio. The velocity dispersion is only recovered well in the $1 \mathrm{~K}$ model.

\subsection{A Self-absorption Model Fit as Optically Thin}

If in reality the HI is self-absorbing, how wrong will models be that assume an optically-thin medium? To test this, we fit the self-absorption models from the previous test with optically-thin models. The results are shown in Figure 6 Again the rotation curve is recovered well out to $12 \mathrm{kpc}$ or so. Clearly, the rotation curve does not suffer much from self-absorption. As expected, the face-on surface density is not recovered well at all, with the highest column densities estimated at only $\sim 70 \%$ of their true values. The disc is consistently estimated to be somewhat thicker than it in reality is. The velocity dispersion is not recovered well at 

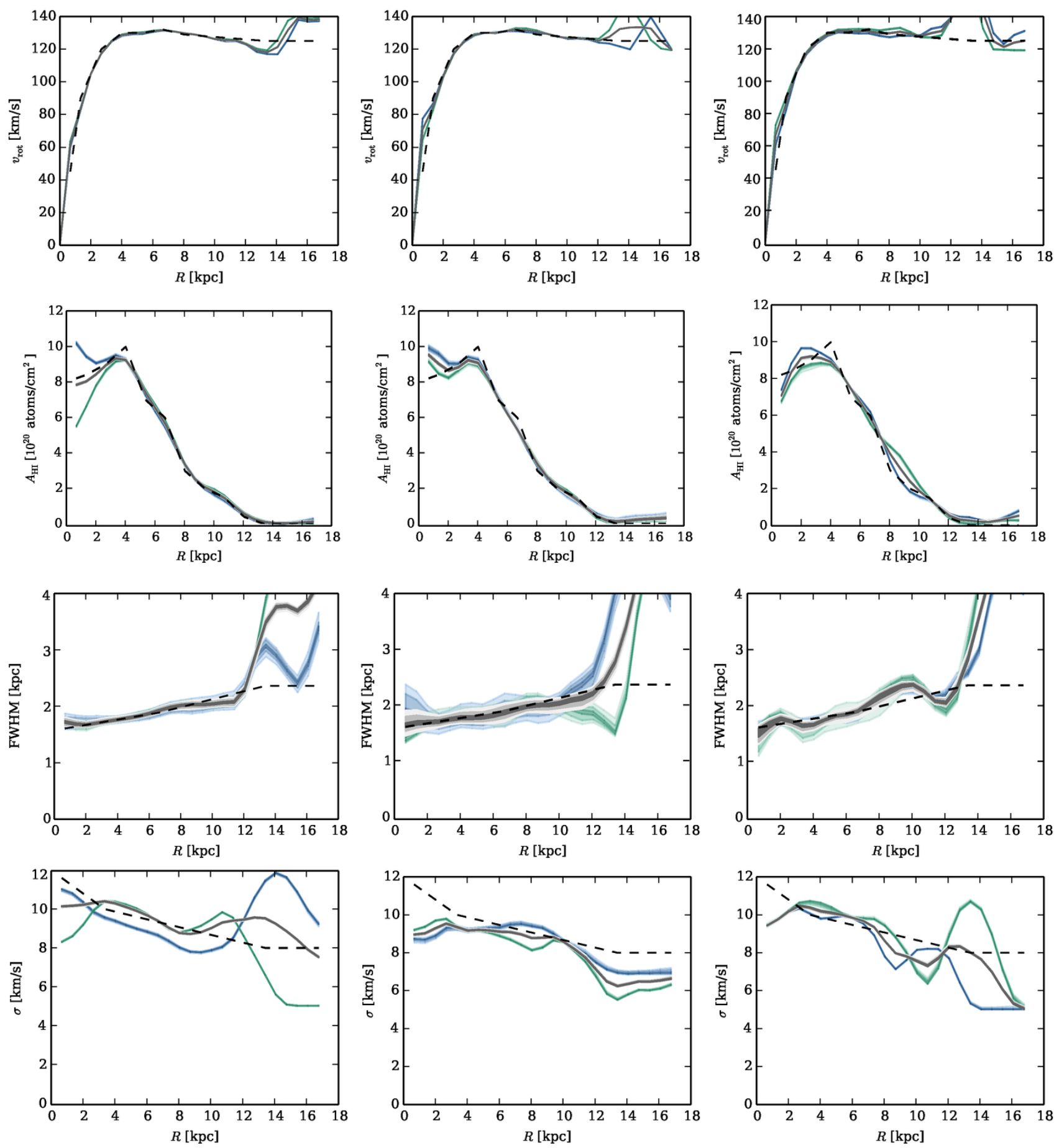

Figure 4. Optically thin test models with noises of $1 \mathrm{~K}, 3 \mathrm{~K}$ and $5 \mathrm{~K}$ (left-to-right). Top panels shows the rotation curve $v_{\text {rot }}$, second row shows the face-on surface density $A_{\mathrm{HI}}$. Third row shows the thickness of the model. Fourth row shows the fit to the velocity dispersion. Green colours denote the left side of the galaxy, blue colours show the right side. The grey band is the combined result. Dashed lines show the true parameters of the model.

all. Performing optically thin fits on self-absorbed distributions produces results significantly in error, even at relatively small radii.

\subsection{A More Complicated Model}

In the previous tests we have modelled and fitted the galaxies at a perfect edge-on inclination of $90^{\circ}$. Our fitting strategy always assumes this perfectly edge-on inclination. As a final test, we examine the quality of the fit if the actual galaxy is not perfectly edge-on, but is at an inclination of $88.8^{\circ}$. We also include a modest side-on warp that begins at 

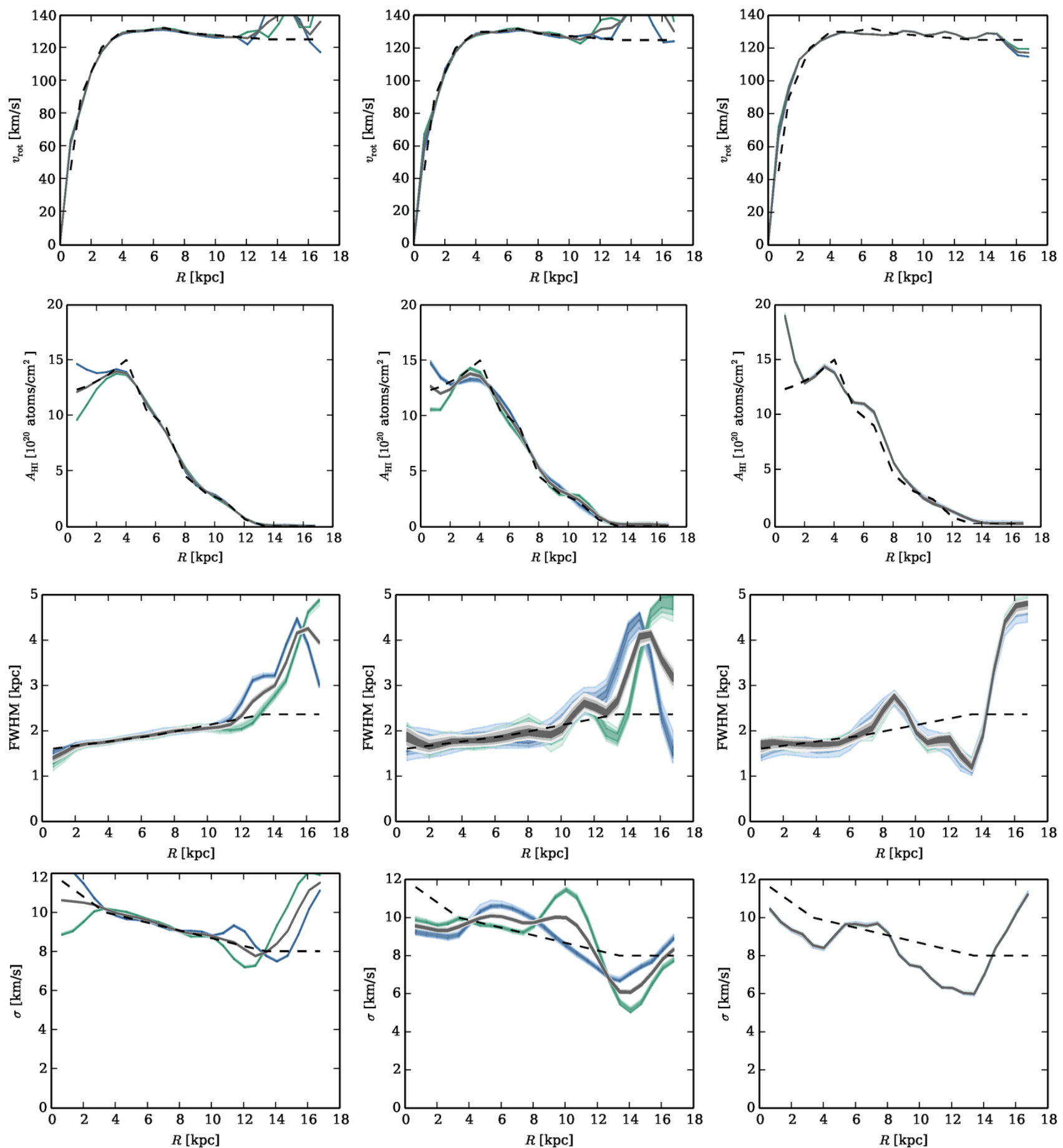

Figure 5. Self-absorption $\left(T_{\text {spin }}=100 \mathrm{~K}\right)$ test models with noises of $1 \mathrm{~K}, 3 \mathrm{~K}$ and $5 \mathrm{~K}$ (left-to-right). Top panels shows the rotation curve $v_{\text {rot }}$, second row shows the face-on surface density $A_{\mathrm{HI}}$. Third row shows the thickness of the model. Fourth row shows the fit to the velocity dispersion. Green colours denote the left side of the galaxy, blue colours show the right side. The grey band is the combined result. Dashed lines show the true parameters of the model.

$R=8 \mathrm{kpc}$. We again run the fits with intrinsic noises of 1,3 and $5 \mathrm{~K}$ and use optically thin models. The results are shown in Figure 7. The true systemic velocity is recovered to within $0.6 \mathrm{~km} / \mathrm{s}$, while the channel width was $3.3 \mathrm{~km} / \mathrm{s}$. The position angle is accurate to within $0.9^{\circ}$. The central position is found to within 0.7". The measurement of the thickness of the disc is wrong beyond a radius of $9 \mathrm{kpc}$, which is due to the onset of the warp. Our conclusions remain the same as the previous tests: All parameters are recovered well at $1 \mathrm{~K}$, but the measurements of the velocity dispersion become increasingly difficult at $3 \mathrm{~K}$ and for all parameters at $5 \mathrm{~K}$. 

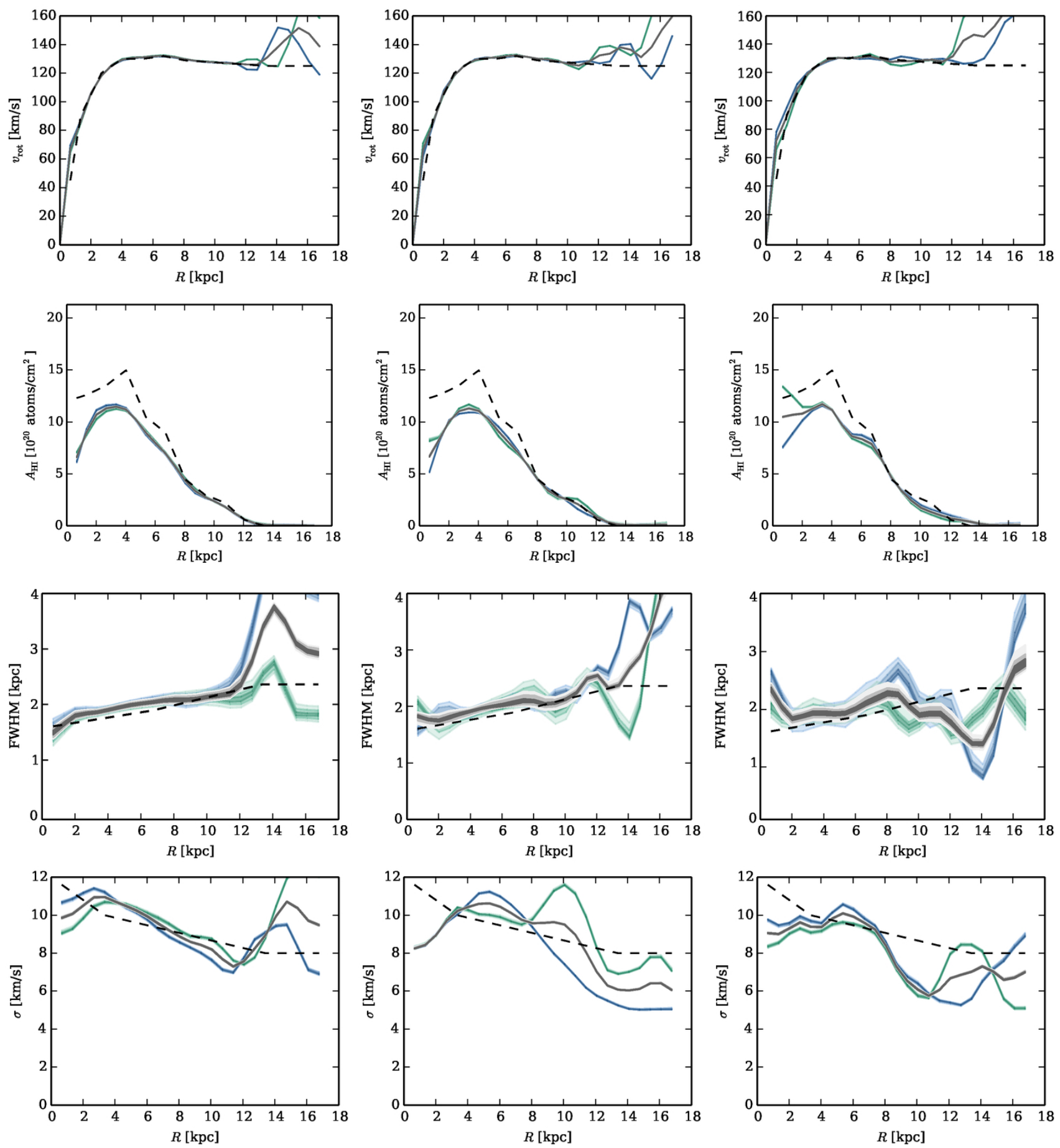

Figure 6. Optically thin fits to self-absorption $\left(T_{\text {spin }}=100 \mathrm{~K}\right)$ test models with noises of $1 \mathrm{~K}, 3 \mathrm{~K}$ and $5 \mathrm{~K}$ (left-to-right). Top panels shows the rotation curve $v_{\text {rot }}$, second row shows the face-on surface density $A_{\mathrm{HI}}$. Third row shows the thickness of the model. Fourth row shows the fit to the velocity dispersion. Green colours denote the left side of the galaxy, blue colours show the right side. The grey band is the combined result. Dashed lines show the true parameters of the model.

\subsection{Concluding Remarks}

So what can we conclude? We have shown that our fitting strategy can recover the parameters in these edge-on galaxies. The rotation curve and the face-on surface density are recovered well in all cases. The biggest problem in fitting these galaxies comes from the velocity dispersion, which can only be fit in very low noise HI data cubes. We will thus adopt a constant velocity dispersion for galaxies where the noise is too high. The flaring will only be problematic in high noise HI data cubes. We note that our strategy produces these results in idealized circumstances. In reality, the galaxies can (and will) have asymmetries, spiral arms, warps, lagging haloes, etc.; which will make our measurements less accurate then the presented test models. 

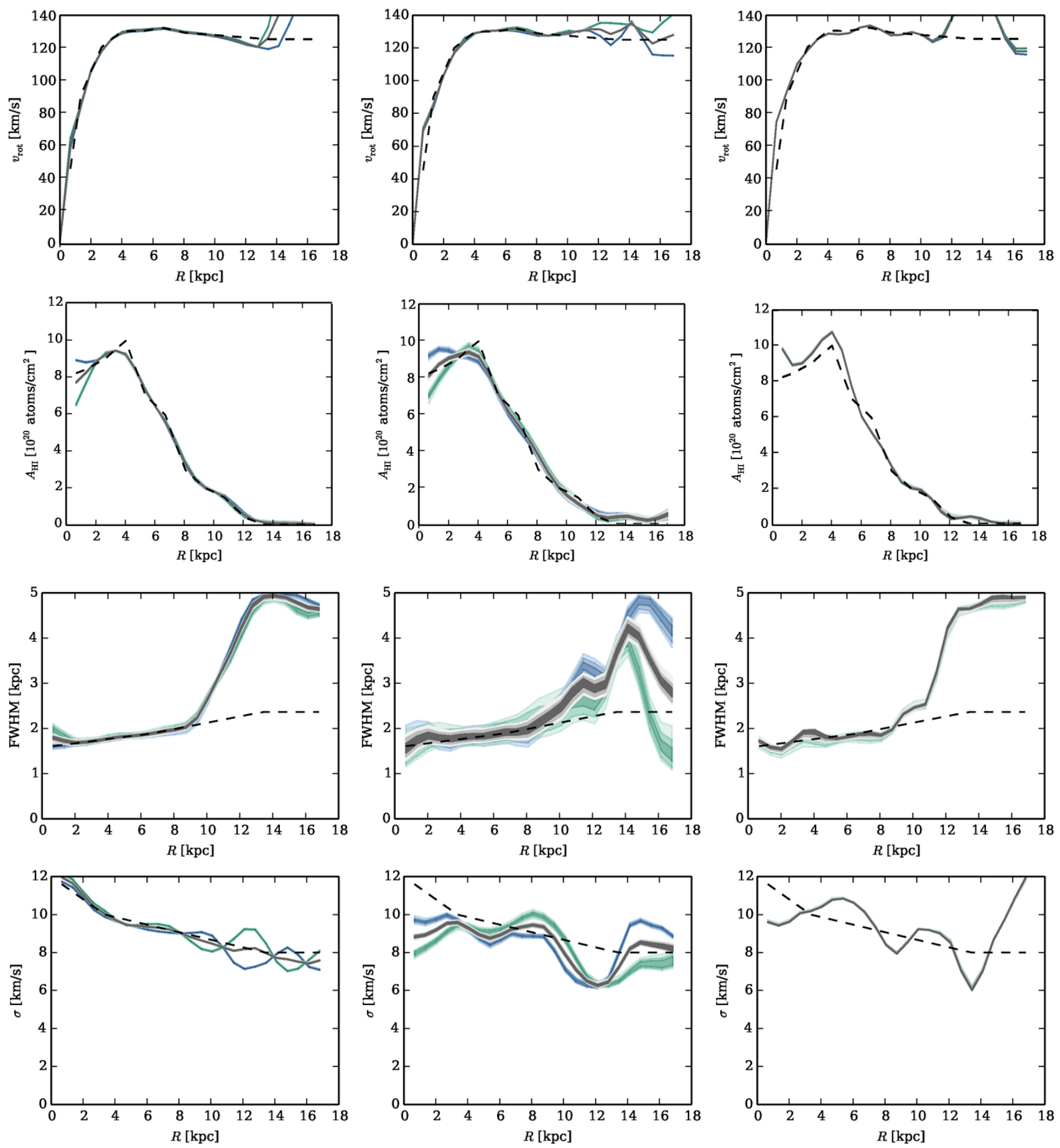

Figure 7. Optically thin test models with noises of $1 \mathrm{~K}, 3 \mathrm{~K}$ and $5 \mathrm{~K}$ (left-to-right). The reference images have an inclination of $88.8^{\circ}$. Top panels shows the rotation curve $v_{\text {rot }}$, second row shows the face-on surface density $A_{\mathrm{HI}}$. Third row shows the thickness of the model. Fourth row shows the fit to the velocity dispersion. Green colours denote the left side of the galaxy, blue colours show the right side. The grey band is the combined result. Dashed lines show the true parameters of the model.

We have also shown that fitting a self-absorption reference model with an optically thin model will lead to bad results. In particular, the face-on surface density will be too low, while the thickness measured will be too high and the velocity dispersion will be wrong. Only the rotation curve will be estimated to a reasonable degree. As we argued before, galaxies are in reality expected to have self-absorbing
HI . This result thus implies that the results found in the literature based on the assumption of zero optical thickness are underestimating the true HI content of galaxies. 


\section{FITS TO ACTUAL GALAXIES}

In Paper I, we have presented the HI observations for eight edge-on galaxies. In this section, we will focus on modelling these galaxies in more detail. We aim to fit the rotation curve, velocity dispersion, thickness and face-on surface density of the galaxies, as we will use these in Paper $\mathrm{V}$ to model the hydrostatics of the gas. Based on the results of the previous section, it is of vital importance to have the lowest noise levels we possibly can. To this end, we re-image the data such that we can still resolve the vertical structure of the disc, but have the lowest noise possible. For more details on the HI reduction, see Section 4.1 of Paper I. In Table 3 of Paper I, we denote the original HI data cubes. IC 5052 has been re-imaged with a FWHM beam of 25 " and a noise $\sigma$ of $1.7 \mathrm{~K}$. ESO 115-G021 now has a FWHM beam of 25" and a noise $\sigma$ of $1.27 \mathrm{~K}$. For ESO 274-G001, we adopt the 30" beam from Table 3 of Paper I, which has a noise $\sigma$ of $1.2 \mathrm{~K}$. UGC 7321 has been re-imaged with a FWHM beam of 25 " and a noise $\sigma$ of $1.7 \mathrm{~K}$. Based on these noise levels and the results of the previous section, we have decided to model UGC 7321, ESO 115-G021 and ESO 274-G001 with the velocity dispersion as a free parameter that can vary with radius. The other galaxies will be modelled using an assumed constant velocity dispersion of $10 \mathrm{~km} / \mathrm{s}$.

We do not include the results for IC 2531 and ESO 146G014. In Section 5.1 of Paper I, we commented on the remarkable thin disc that IC 2531 appeared to have. This disc, combined with the high noise, made it impossible to measure the thickness of the HI disc accurately. We were unable to make a reliable fit to ESO 146-G014. As we discussed in Section 5.6 of Paper I, the galaxy probably has a warp and is not sufficiently edge-on for our measurements.

\section{$4.1 \quad$ IC 5052}

Galaxy IC 5052 has proven hard to model. We show both the optically thin model and the self-absorption model in Figure 8. As we noted in Section 5.2 of Paper I, the galaxy has a very strong warp. Looking back at Figure 3 in Paper I, we can see that the onset of the warp occurs just beyond that same radius (180"). This warp is responsible for the very large thickness that is found beyond $5 \mathrm{kpc}$. The declining rotation curve beyond $5 \mathrm{kpc}$ is also due to this component.

The self-absorption models have a total mass of $9.5 \pm$ $0.9 \times 10^{8} \mathrm{M}_{\odot}$, while the optically thin model recovers only $7.4 \pm 0.7 \times 10^{8} \mathrm{M}_{\odot}$. This is less than our initial estimate of $8.9 \times 10^{8} \mathrm{M}_{\odot}$ in Table 4 of Paper I. This trend is also visible in the other galaxies. It is because the fit can only model a smooth medium. Local bright regions in the observations therefore cannot be accurately recovered. Any warp will also harbor mass, which is not recovered here. Comparing the two models (and ignoring the right-hand selfabsorption model), the optically thin model is missing about a quarter of the HI .

O'Brien et al. (2010a) also had trouble with modelling IC 5052. They did not resolve the warp, but found clear evidence for asymmetry. Comparing the rotation curves to our work, the results on both sides look similar up to $6 \mathrm{kpc}$, but subsequent downturn is not detected as strongly in their work. The thickness estimates are similar. Their face-on column density is beyond $2 \mathrm{kpc}$ similar to our optically thin re- sult, although due to our smoothing the data is less bumpy. The inner parts are different, but are not well resolved in our models. The results we find for the flaring beyond a radius of $5 \mathrm{kpc}$ are doubtful. We have chosen in Paper $\mathrm{V}$ in this series where we analyze our data further not to consider this galaxy.

\section{$4.2 \quad$ IC 5249}

The HI structure and kinematics of galaxy IC 5249 have been previously analyzed by Abe et al. (1999), van der Kruit et al. (2001) and O'Brien et al. (2010a). Abe et al. (1999) report a linearly rising rotation curve to about $100 \mathrm{~km} / \mathrm{s}$ at $17 \mathrm{kpc}$. The data was re-analyzed by van der Kruit et al. (2001), who reports a steeper inner rotation curve that flattens out to $105 \mathrm{~km} / \mathrm{s}$ at $10 \mathrm{kpc}$ and remains flat throughout the remaining disc. This rotation curve was confirmed by O'Brien et al. (2010a). Our fits with both the optically thin model and the self-absorption model confirms their results (Figure 9). We however note that rotation curves differs between both sides, so the galaxy might be asymmetric.

Comparing the face-on surface densities, our optically thin fit looks very similar to the observed face-on density by van der Kruit et al. (2001). The peak surface density occurs at a radius of $17-18 \mathrm{kpc}$ at a value of $5.8 \times 10^{20}$ atoms $/ \mathrm{cm}^{2}$. More inward, the profile has a lower density. We also reproduce their dip in the face-on profile at $\sim 11 \mathrm{kpc}$. The face-on surface density of O'Brien et al. (2010a) looks similar to our left-side profile. Their joined profile does recover the peak at $17-18 \mathrm{kpc}$, but does not have the lower central densities. Our self-absorption fit finds more HI . The peak at $17-18 \mathrm{kpc}$ now becomes a plateau near at $8 \times 10^{20}$ atoms $/ \mathrm{cm}^{2}$ (30\% higher). The profile also shows the dip near $11 \mathrm{kpc}$, although the average inner density remains far larger than the optically thin result. The inner parts of the optically thin model are 'saturated' by the HI from outer radii, and the model compensates for this by lowering the face-on column densities in the inner parts.

We find a strongly flaring disc in both fits, with an inner thickness of only $500 \mathrm{pc}$, but increasing linearly with radius to nearly $2.6 \mathrm{kpc}$ at $25 \mathrm{kpc}$. In Paper $\mathrm{V}$ we will only use the flaring out to $14 \mathrm{kpc}$ for the fits to determine the axis ratio of the dark matter halo. This is different than O'Brien et al. (2010a), who finds the same linearly increasing thickness, but only going out to $1.5 \mathrm{kpc}$ thickness at a radius of $20 \mathrm{kpc}$. An average thickness of $1.1 \pm 0.3 \mathrm{kpc}$ was reported by van der Kruit et al. (2001), although their Figure 6 can be seen to increase linearly to a thickness of $3.3 \mathrm{kpc}$ at $14 \mathrm{kpc}$. Given their error bars, this result is consistent with our findings.

Comparing the total masses of both types of fits, we find that the optically thin model has a total HI mass of $4.8 \pm 0.2 \times 10^{9} \mathrm{M}_{\odot}$. The self-absorption model finds a total of $7.8 \pm 0.8 \times 10^{9} \mathrm{M}_{\odot}$. This would put the hidden HI mass fraction at about a third. The optically thin mass is however significantly lower than our initial estimate of $5.6 \times 10^{9} \mathrm{M}_{\odot}$ (Table 4 of Paper I).

\subsection{ESO 115-G021}

Galaxy ESO 115-G021 is a symmetric, slow rotating galaxy which we have managed to fit well (see Figures 10 and 11 . 

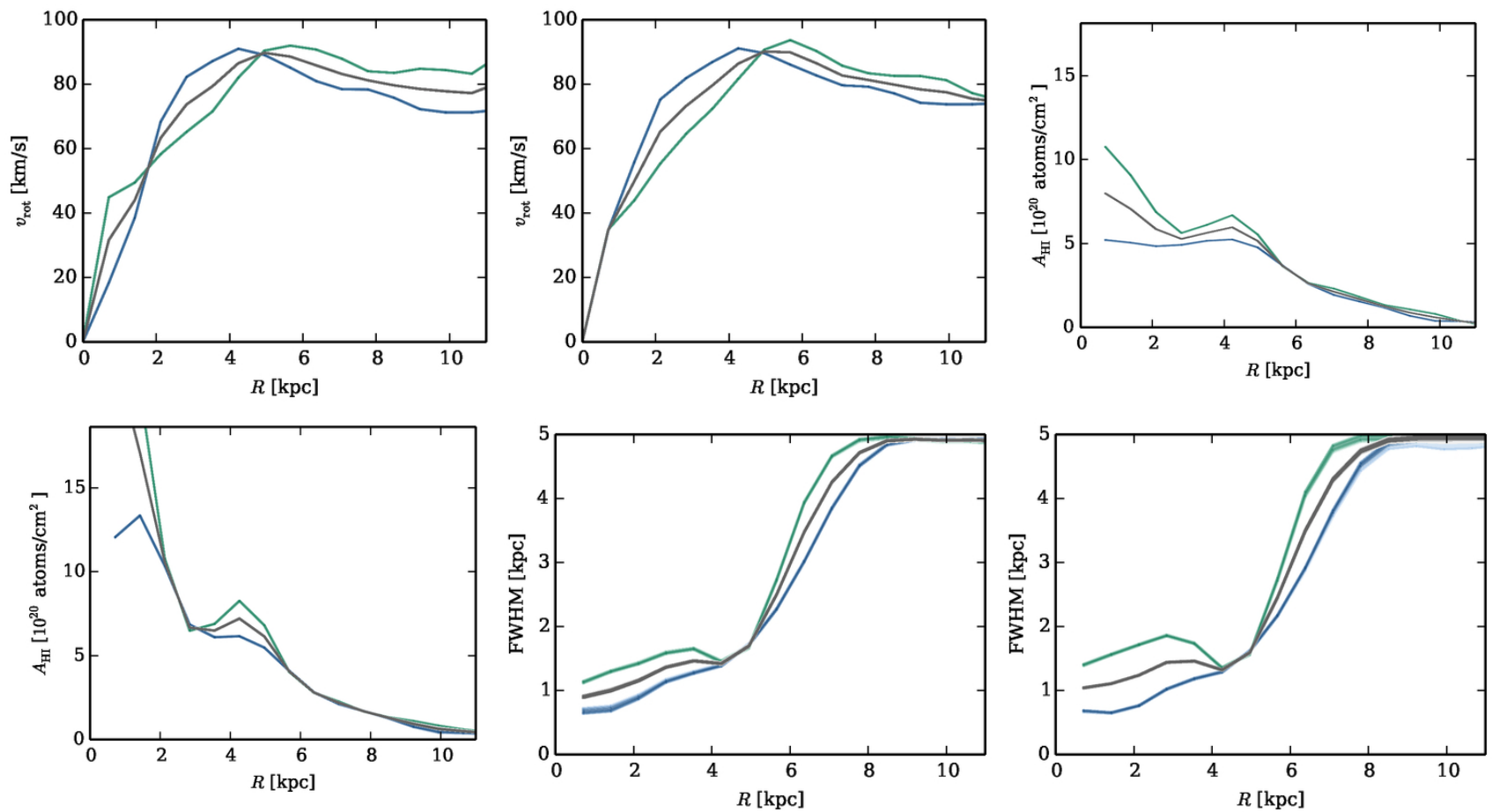

Figure 8. Decomposition results for IC 5052 assuming an optically thin mode (left column) and self-absorbing HI medium (right column). Colour ranges have been chosen such that they contain $68 \%, 95 \%$ and $99.7 \%$ of the distribution. Assuming a normal distribution, this represents the 1,2 and $3 \sigma$ dispersion from the mean. Green lines are for the left side of the galaxy, cyan lines for the right side.
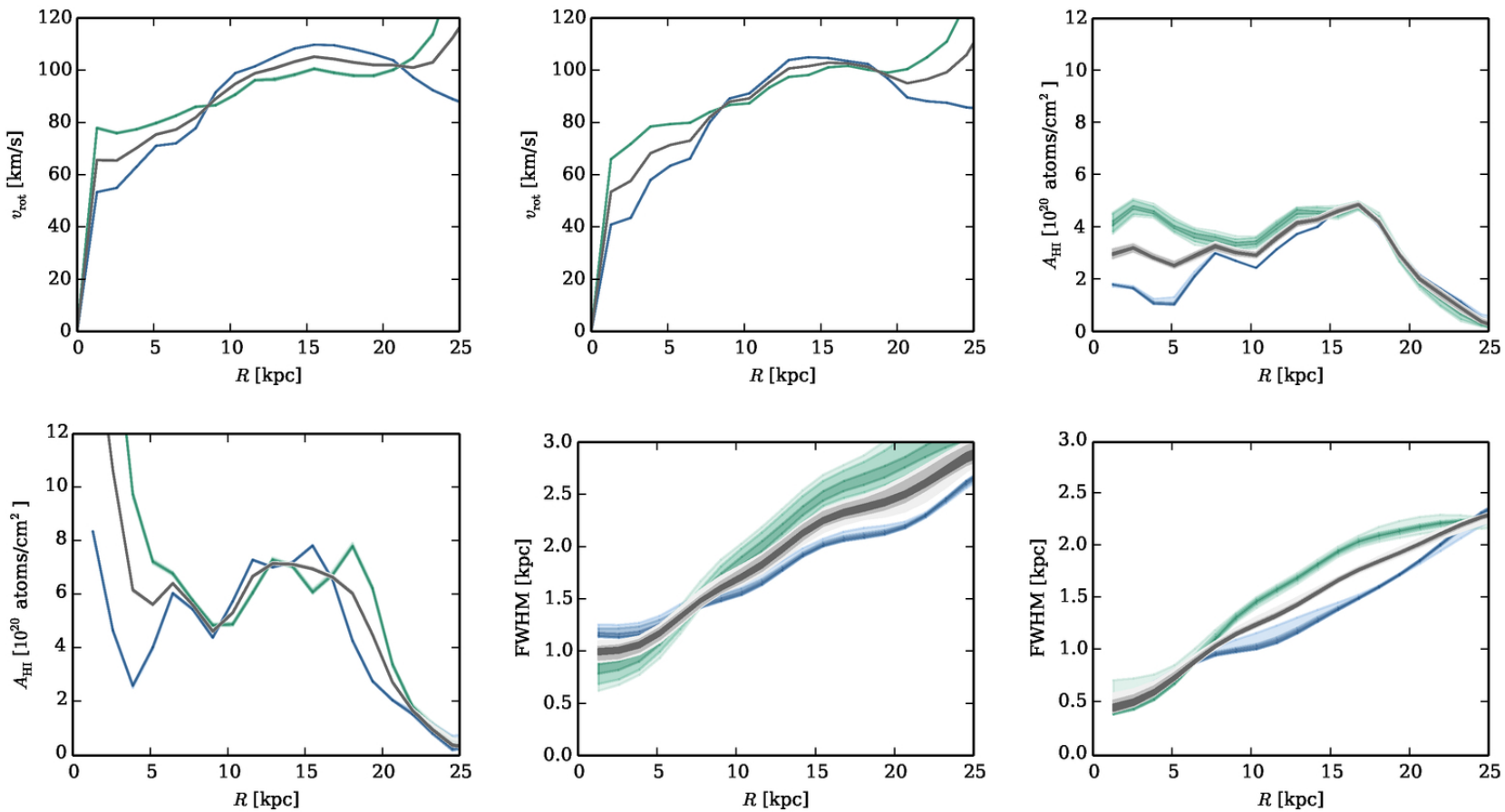

Figure 9. Decomposition results for IC 5249 assuming an optically thin mode (left column) and self-absorbing HI medium (right column). Colour ranges have been chosen such that they contain $68 \%, 95 \%$ and $99.7 \%$ of the distribution. Assuming a normal distribution, this represents the 1,2 and $3 \sigma$ dispersion from the mean. Green lines are for the left side of the galaxy, cyan lines for the right side. The grey band is the combined result. 

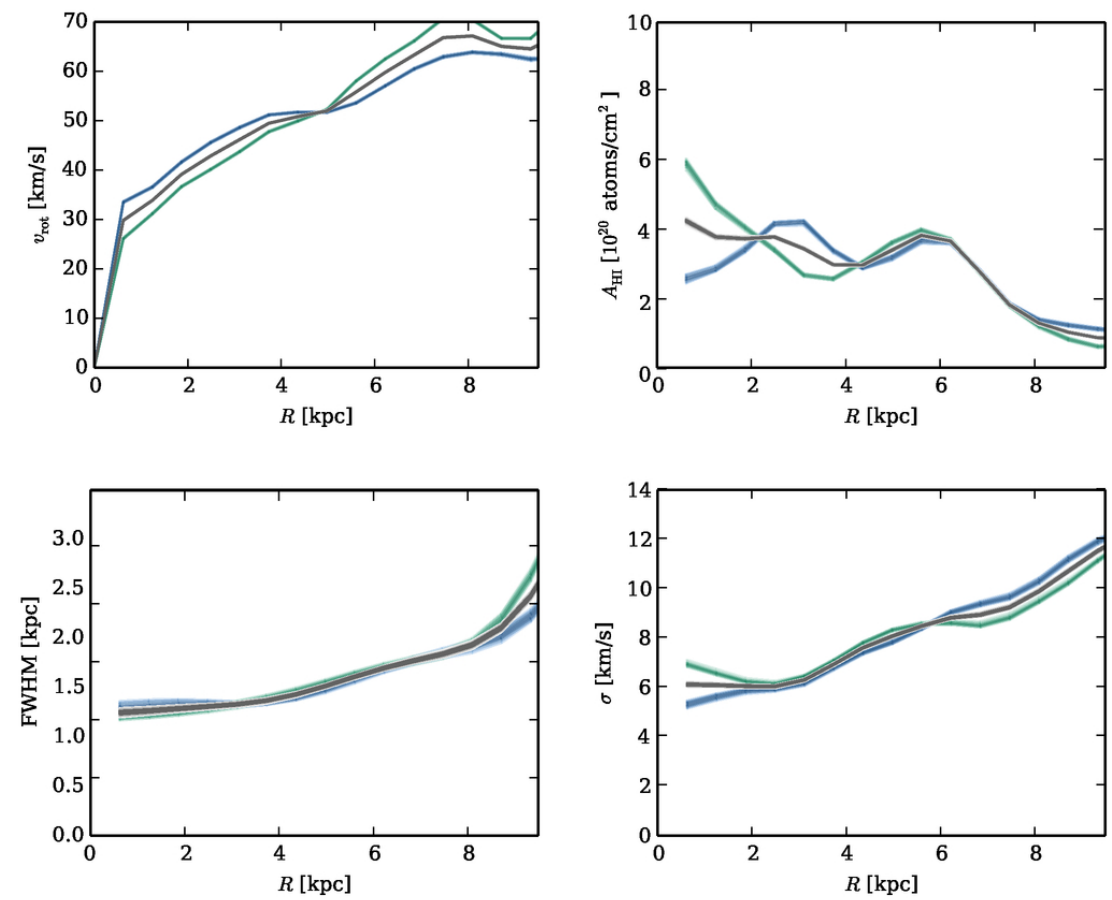

Figure 10. Decomposition results for ESO 115-G021 assuming an optically thin model. Colour ranges have been chosen such that they contain $68 \%, 95 \%$ and $99.7 \%$ of the distribution. Assuming a normal distribution, this represents the 1,2 and $3 \sigma$ dispersion from the mean. Green lines are for the left side of the galaxy, cyan lines for the right side. The grey band is the combined result.
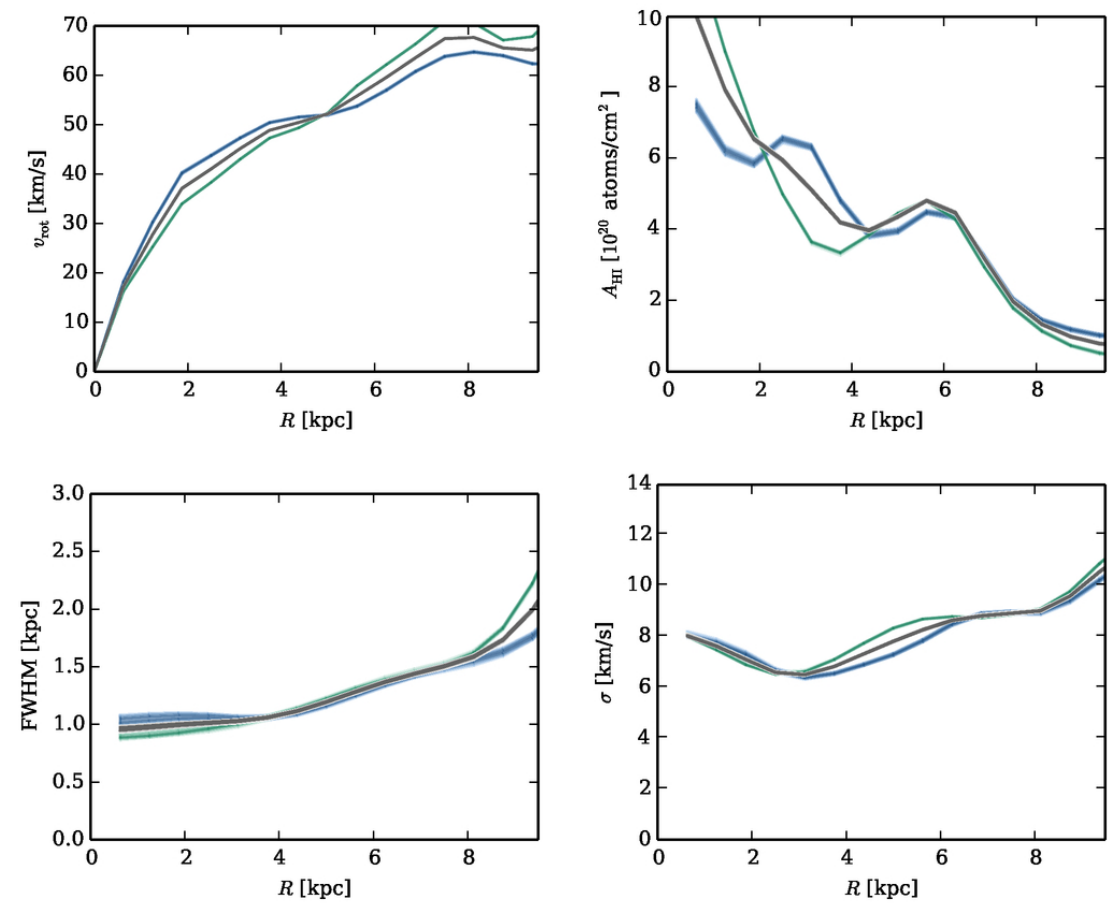

Figure 11. Decomposition results for ESO 115-G021 assuming a self-absorption model. Colour ranges have been chosen such that they contain $68 \%, 95 \%$ and $99.7 \%$ of the distribution. Assuming a normal distribution, this represents the 1, 2 and $3 \sigma$ dispersion from the mean. Green lines are for the left side of the galaxy, cyan lines for the right side. The grey band is the combined result. 

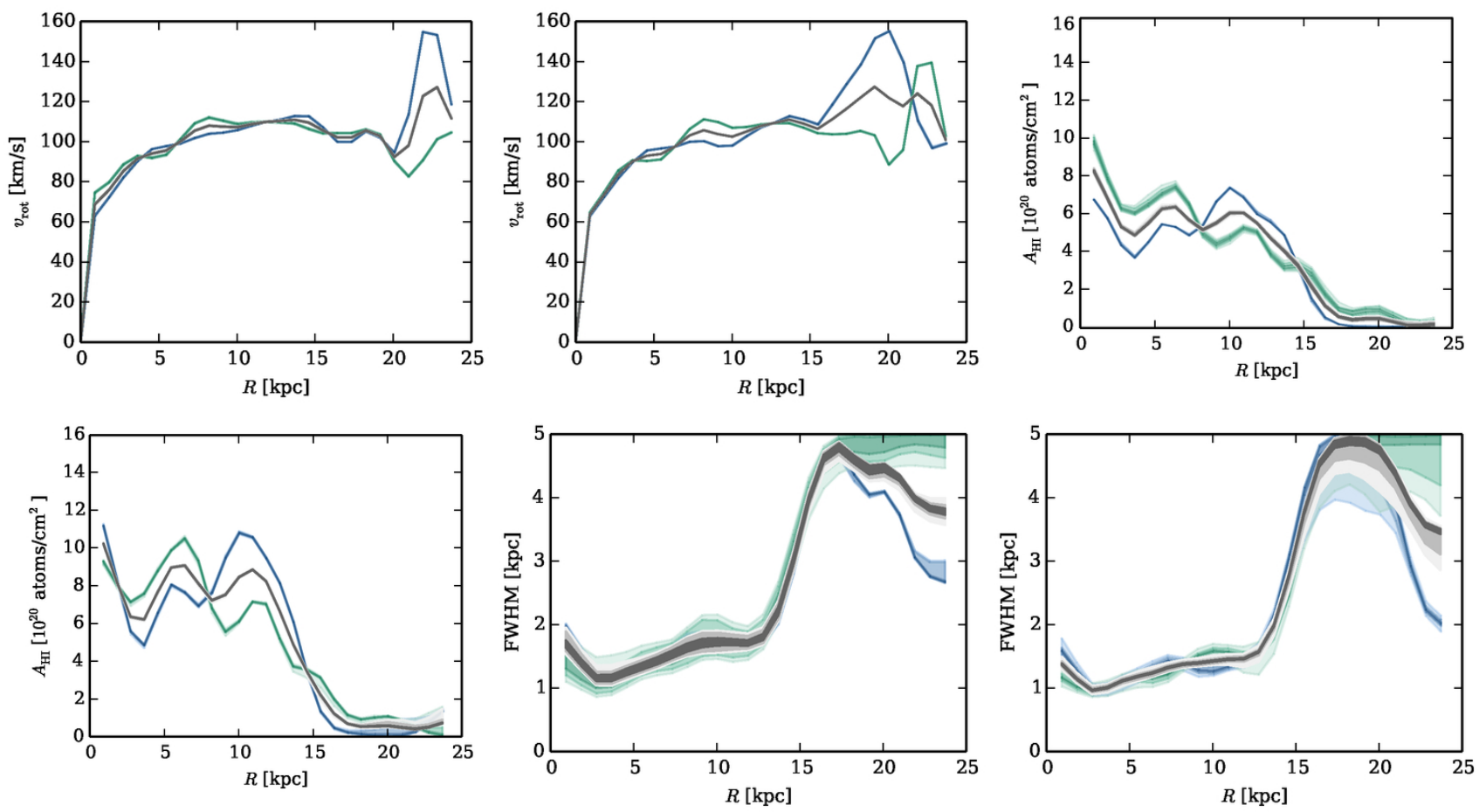

Figure 12. Decomposition results for ESO 138-G014 assuming an optically thin mode (left column) and self-absorbing HI medium (right column). Colour ranges have been chosen such that they contain $68 \%, 95 \%$ and $99.7 \%$ of the distribution. Assuming a normal distribution, this represents the 1,2 and $3 \sigma$ dispersion from the mean. Green lines are for the left side of the galaxy, cyan lines for the right side. The grey band is the combined result.

The rotation curve rises from an initial $30 \mathrm{~km} / \mathrm{s}$ in the inner parts almost linearly to $65 \mathrm{~km} / \mathrm{s}$ around $8 \mathrm{~km} / \mathrm{s}$. The only other analysis of the rotation curve of this galaxy is by O'Brien et al. (2010a), who report a very similar rotation curve. The face-on surface density of our optically thin model is also similar to theirs: A plateau between $3-5 \times 10^{20}$ atoms $/ \mathrm{cm}^{2}$ and then rapidly dropping off. The self-absorption fit to the surface density yields a similar profile, although the inner part has a bit more HI .

The thickness of this galaxy is remarkably well behaved and consistent between both fits. Starting near $700 \mathrm{pc}$ in the inner parts, it rises linearly to $1.5 \mathrm{kpc}$ at a radius of $8 \mathrm{kpc}$. This is different to O'Brien et al. (2010a), who report a stronger increase to over $3 \mathrm{kpc}$ beyond a radius of $6 \mathrm{kpc}$, although they note they had insufficient available positions in their data for a proper fit.

The velocity dispersion fit to the optically thin model is surprising; the galaxy appears to have an increasing velocity dispersion, which is $7 \mathrm{~km} / \mathrm{s}$ in the inner parts and $10 \mathrm{~km} / \mathrm{s}$ at $6 \mathrm{kpc}$. The self-absorption velocity dispersion is more stable, although it is still varying a bit. This behavior is likely due to noise in the data.

Comparing the total masses, the optically thin model yields $5.6 \pm 0.2 \times 10^{9} \mathrm{M}_{\odot}$ and $7.2 \pm 0.1 \times 10^{9} \mathrm{M}_{\odot}$ for the selfabsorption model. Thus about a quarter of the HI is hidden in the model.

\subsection{ESO 138-G014}

Galaxy ESO 138-G014 has been well resolved. The optically thin model and the self-absorption model are shown in Fig- ure 12 The rotation curve rises steeply to $70 \mathrm{~km} / \mathrm{s}$ in the inner parts, and gradually increase to a maximum of $105 \mathrm{~km} / \mathrm{s}$ near $8 \mathrm{kpc}$. Beyond this radius, it levels off. The curve is very similar to that measured by O'Brien et al. (2010a).

The disc is reasonably thick, starting at $1.1 \mathrm{kpc}$ near $2.5 \mathrm{kpc}$ radius and increasing linearly towards $1.8 \mathrm{kpc}$ at $9 \mathrm{kpc}$ (for the optically thin model). In the self-absorption model, the disc is less thick, starting around $1 \mathrm{kpc}$ in the inner parts and increasing only to $1.5 \mathrm{kpc}$ at $9 \mathrm{kpc}$. In both models, around $12-13 \mathrm{kpc}$ the thickness suddenly increases drastically, almost certainly due to the warp seen in the total HI map (Fig. 7 in Paper I). Note that the increase towards a thickness of $2 \mathrm{kpc}$ in the centre is unreliable. O'Brien et al. (2010a) do not trust their thickness measurements and so we cannot compare their results with ours. In Paper V we will only use the flaring out to $6 \mathrm{kpc}$ for the fits to determine the axis ratio of the dark matter halo.

In the optically thin model, the face-on surface density shows a clear plateau between 5 and $6 \times 10^{20}$ atoms $/ \mathrm{cm}^{2}$ up to a radius of $12-13 \mathrm{kpc}$, and dropping off rapidly beyond that. The self-absorption model results in more HI, with the plateau at a higher $6-9 \times 10^{20}$ atoms $/ \mathrm{cm}^{2}$. The (optically thin) model of O'Brien et al. (2010a) also finds a plateau near $6 \times 10^{20}$ atoms $/ \mathrm{cm}^{2}$, although it is higher near the inner parts.

Comparing the two mass estimates, our optically thin model has a total mass of $3.4 \pm 0.1 \times 10^{9} \mathrm{M}_{\odot}$, while our selfabsorption model finds $4.6 \pm 0.2 \times 10^{9} \mathrm{M} \odot$. The total hidden HI fraction is thus $28 \pm 1 \%$ of the total HI . 

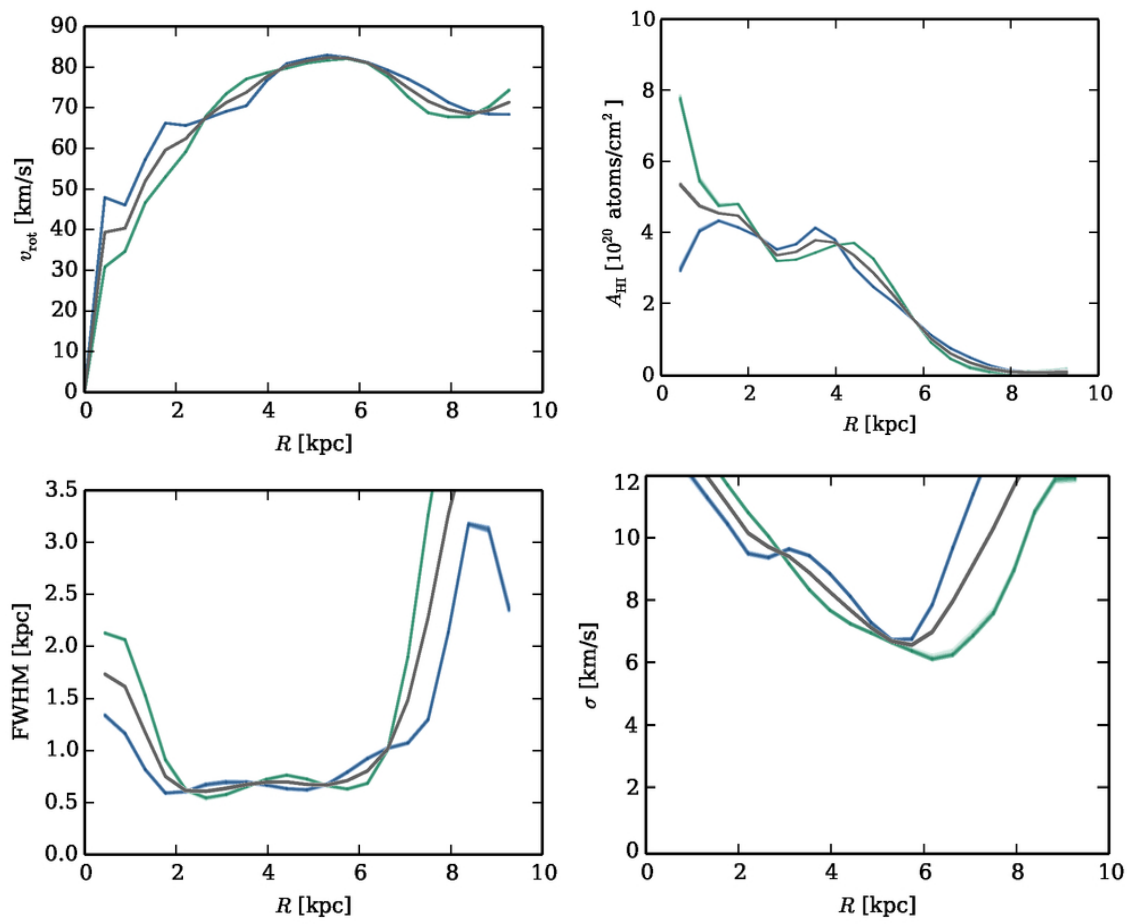

Figure 13. Decomposition results for ESO 274-G001 assuming an optically thin model. Colour ranges have been chosen such that they contain $68 \%, 95 \%$ and $99.7 \%$ of the distribution. Assuming a normal distribution, this represents the 1, 2 and $3 \sigma$ dispersion from the mean. Green lines are for the left side of the galaxy, cyan lines for the right side. The grey band is the combined result.
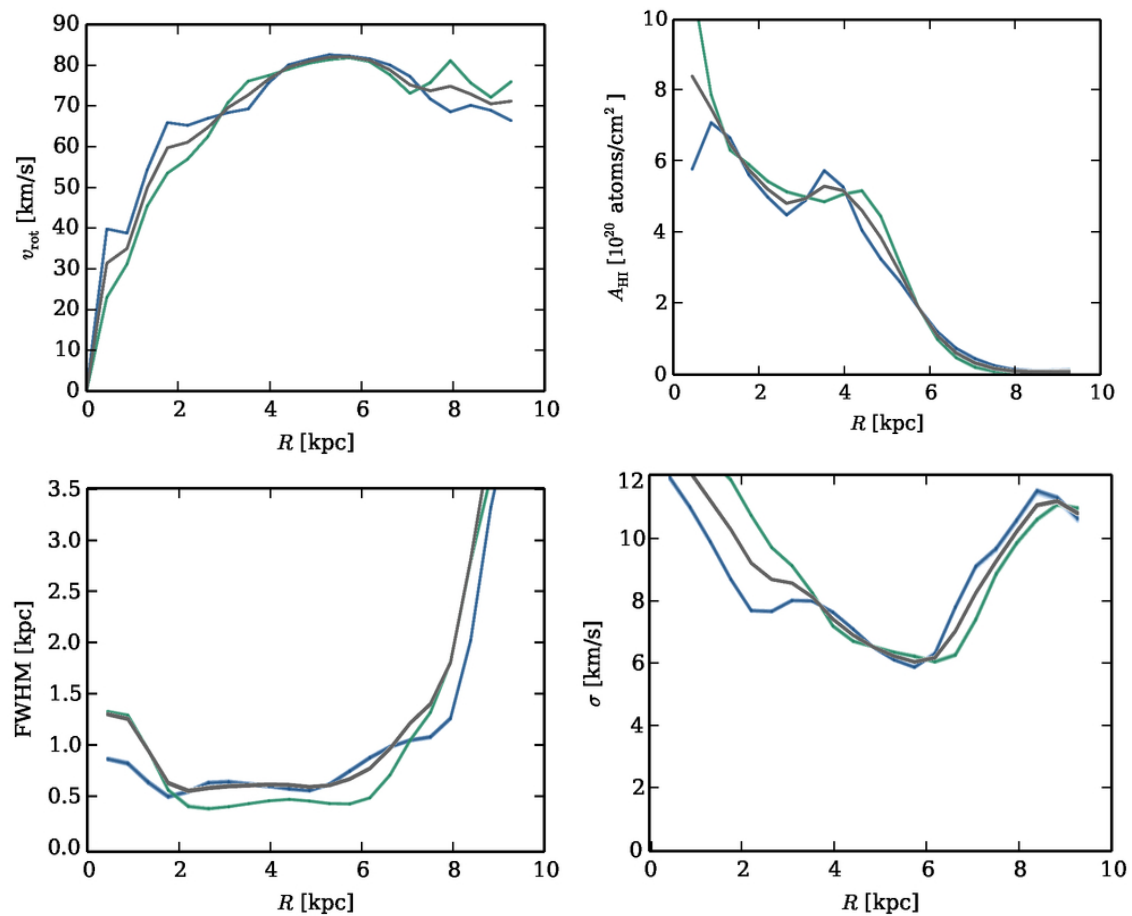

Figure 14. Decomposition results for ESO 274-G001 assuming a self-absorption model. Colour ranges have been chosen such that they contain $68 \%, 95 \%$ and $99.7 \%$ of the distribution. Assuming a normal distribution, this represents the 1, 2 and $3 \sigma$ dispersion from the mean. Green lines are for the left side of the galaxy, cyan lines for the right side. The grey band is the combined result. 

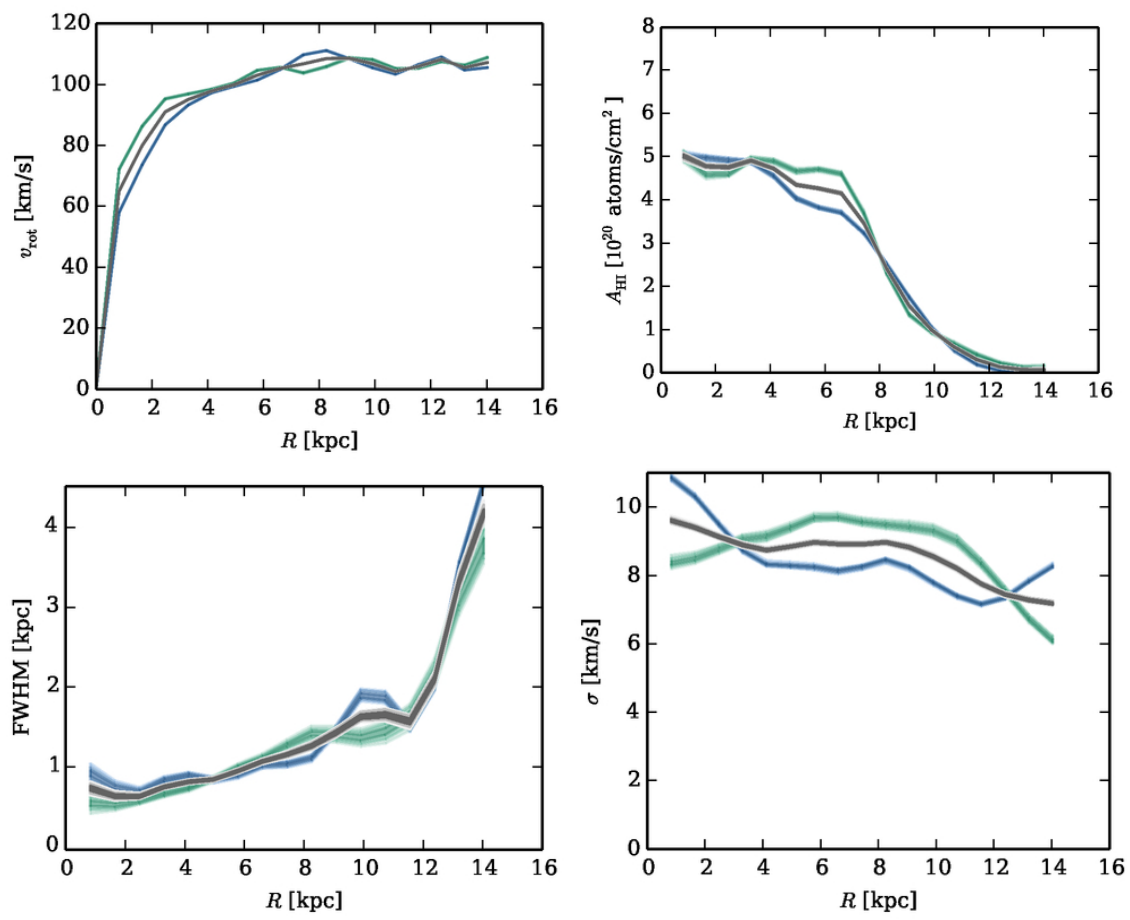

Figure 15. Decomposition results for UGC 7321 assuming an optically thin model. Colour ranges have been chosen such that they contain $68 \%, 95 \%$ and $99.7 \%$ of the distribution. Assuming a normal distribution, this represents the 1, 2 and $3 \sigma$ dispersion from the mean. Green lines are for the left side of the galaxy, cyan lines for the right side. The grey band is the combined result.
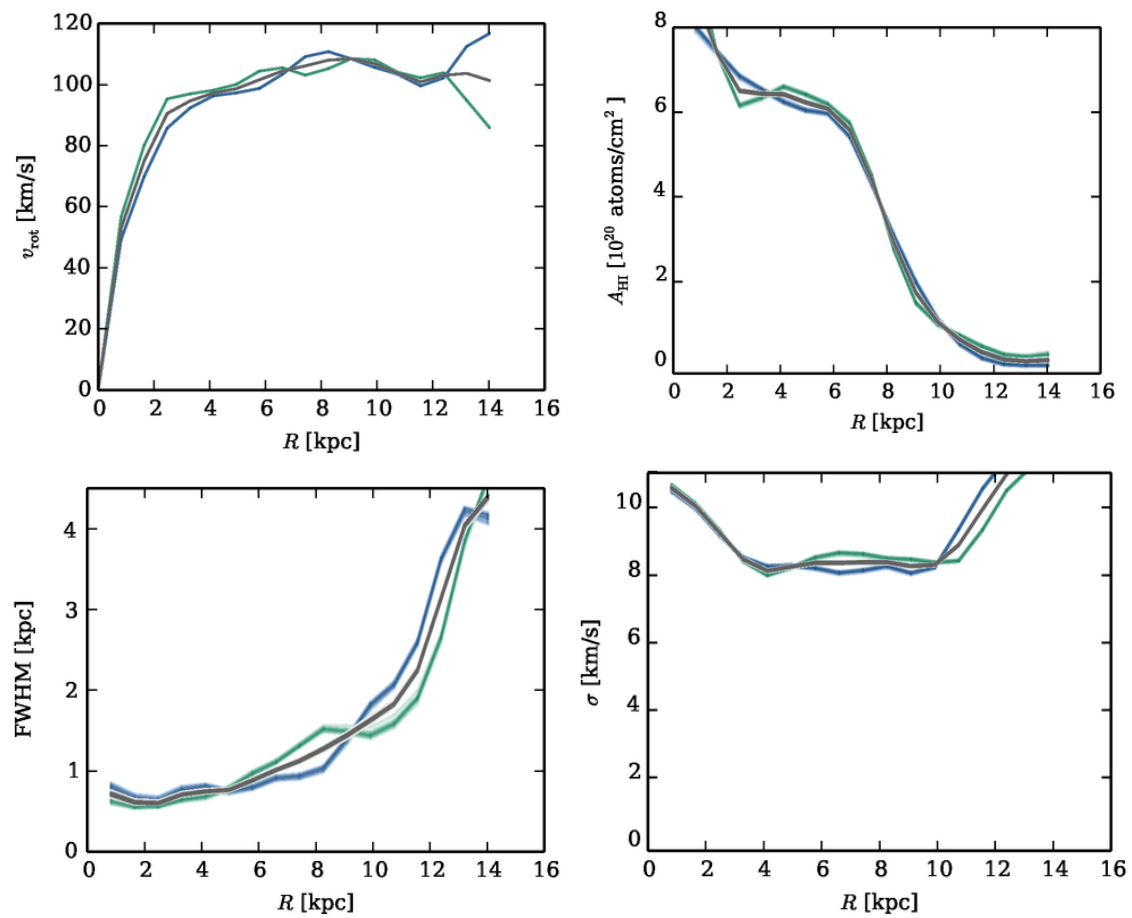

Figure 16. Decomposition results for UGC 7321 assuming a self-absorption model. Colour ranges have been chosen such that they contain $68 \%, 95 \%$ and $99.7 \%$ of the distribution. Assuming a normal distribution, this represents the 1,2 and $3 \sigma$ dispersion from the mean. Green lines are for the left side of the galaxy, cyan lines for the right side. The grey band is the combined result. 


\subsection{ESO 274-G001}

As we discussed in Section 5.7 in Paper I, galaxy ESO 274G001 has a strong continuum source at its central position. This causes strong HI absorption, leading to our inability to fit the central radii of the galaxy accurately. We trust our results beyond $1.5 \mathrm{kpc}$. We show our optically thin and self-absorption fits in Figures 13 and 14.

The rotation curve of the galaxy starts near $30 \mathrm{~km} / \mathrm{s}$ in the inner parts and rises continuously towards a maximum of $80 \mathrm{~km} / \mathrm{s}$ at $5 \mathrm{kpc}$. A similar result was found by O'Brien et al. (2010a).

The face-on surface density profile of the optically thin model hovers between 3 and $5 \times 10^{20}$ atoms $/ \mathrm{cm}^{2}$, with an average around $3.7 \times 10^{20}$ atoms $/ \mathrm{cm}^{2}$. The face-on surface density profile of the self-absorption model has more HI , in a plateau around $5 \times 10^{20}$ atoms $/ \mathrm{cm}^{2}$ up to $5 \mathrm{kpc}$. In both models, the HI density drops rapidly towards zero at $8 \mathrm{kpc}$. The (optically thin) face-on surface density model of O'Brien et al. (2010a) yielded a similar result as our optically thin model.

With the exception of the inner part, the thickness of the optically thin galaxy starts at $600 \mathrm{pc}$ at $2 \mathrm{kpc}$ radius, and increases to $750 \mathrm{pc}$ at a radius of $6 \mathrm{kpc}$. Similar to previous galaxies, the self-absorption yields a less thick disc, starting at $550 \mathrm{pc}$ at $2 \mathrm{kpc}$, but only increasing to $600 \mathrm{pc}$ at $6 \mathrm{kpc}$ radius. Beyond $6 \mathrm{kpc}$, both models yield a very large thickness, although the HI has dropped to almost zero at those radii. The flaring is very different from that measured by O'Brien et al. (2010a), whom found $100 \mathrm{pc}$ near the inner parts and flaring out linearly towards $1.8 \mathrm{kpc}$ in the outer parts.

Both our models find a velocity dispersion that is dropping with radius. The optically thin model starts near $13 \mathrm{~km} / \mathrm{s}$ and drops towards $7 \mathrm{~km} / \mathrm{s}$ at $6 \mathrm{kpc}$. The selfabsorption model drops towards $6 \mathrm{~km} / \mathrm{s}$ at that radius. Beyond this radius, the velocity dispersion is unreliable. The velocity dispersion measured by O'Brien et al. (2010a) shows a very different behavior, staying constant around $6.5 \mathrm{~km} / \mathrm{s}$ up to $6 \mathrm{kpc}$.

The optically thin model has a total HI mass of $3.1 \pm$ $0.02 \times 10^{8} \mathrm{M}_{\odot}$. The self-absorption model has a total mass of $4.1 \pm 0.1 \times 10^{8} \mathrm{M}_{\odot}$. A total of $25 \pm 1 \%$ of the HI is thus hidden by self-absorption.

\subsection{UGC 7321}

The HI of galaxy UGC 7321 has been previously modelled by Uson \& Matthews (2003) and O'Brien et al. (2010). Matthews \& Wood (2003) investigated the high-latitude HI of the galaxy is detail. The galaxy is the only in our sample which has been observed using the VLA. Together with ESO 274-G001, the galaxy has the highest signal to noise in our sample. We show the results for the fits in Figures 15 and 16

The rotation curve rises steeply in the inner parts, only to level of around $9 \mathrm{kpc}$ at $110 \mathrm{~km} / \mathrm{s}$. A similar behavior was reported by Uson \& Matthews (2003) and O'Brien et al. (2010).

The thickness of the disc starts, in the optically thin model, near $750 \mathrm{pc}$ and increases linearly towards $1.5 \mathrm{kpc}$ at a radius of $10 \mathrm{kpc}$. Beyond this radius, the thickness increases rapidly, due to the presence of a warp. Uson \&
Matthews (2003) estimate the onset of the warp at 150" $(7.2 \mathrm{kpc})$, although it becomes stronger beyond this radius. Our self-absorption fit shows a different profile than the optically thin model. Between zero and five kpc, the thickness is nearly constant at $600-700 \mathrm{pc}$, and only then flares out to $1.5 \mathrm{kpc}$ at $10 \mathrm{kpc}$ radius. In Paper $\mathrm{V}$ we will only use the flaring out to $7.5 \mathrm{kpc}$ for the fits to determine the axis ratio of the dark matter halo.

Both the optically thin model and the self-absorption model find a plateau in the face-on surface density profile. This plateau remains constant out to roughly $7 \mathrm{kpc}$, between 4 and $5 \times 10^{20}$ atoms $/ \mathrm{cm}^{2}$ in the optically thin model, and 6 and $7 \times 10^{20}$ atoms $/ \mathrm{cm}^{2}$ in the self-absorption model. The optically thin results are consistent with both Uson \& Matthews (2003) and O'Brien et al. (2010).

The high quality of the data has also allowed us to fit the velocity dispersion. The optically thin model hovers between 9 and $10 \mathrm{~km} / \mathrm{s}$ up to $10 \mathrm{kpc}$ radius. The self-absorption model yields a decrease from 10 to $8 \mathrm{~km} / \mathrm{s}$ in the first $3 \mathrm{kpc}$, but remains flat at $8 \mathrm{~km} / \mathrm{s}$ up to $10 \mathrm{kpc}$ radius.

The optically thin model has a total mass of $8.9 \pm 0.4 \times$ $10^{8} \mathrm{M}_{\odot}$, while the self-absorption model has a total mass of $1.17 \pm 0.02 \times 10^{9} \mathrm{M}_{\odot}$. A total of $24 \pm 1 \%$ of the HI is thus hidden by self-absorption.

\subsection{Variations of the Velocity Dispersion with Height}

One of the (many) assumptions required for modelling the hydrostatics in a galaxy, is that the HI velocity dispersion is constant with height (isothermal) and only varies with radius. This has been assumed for a very long time, as for example by van der Kruit (1981), based on the Galactic work done by Celnik et al. (1979), who found little evidence for variations. To conclude this paper, we test this assumption. We use the highly resolved observation of ESO 274-G001. We begin with the self-absorption results from Section 4.5 and fix the flaring at the measured values. A mask was created on the central plane of the galaxy with a thickness of $290 \mathrm{pc}$. Two models were created, one in which only this central plane is visible, and one in which this plane is masked. The results are shown in Figure 17. The rotation curve of the high latitude fit can be seen to lag compared to the combined and main profiles. The velocity dispersion of the high latitude fit is $1 \mathrm{~km} / \mathrm{s}$ larger than the central plane fit, with the combined fit falling in between the other two curves.

Note that this test is stretching the data to its absolute limits. The error-bars are large; the only consolation is that we observe this behavior in the fits to the individual sides. These results do not rule out that the HI in galaxies is not isothermal. It is beyond the scope of this project and the available data to test this in more detail in this series of papers. It will be very interesting to explore this using the next generation of radio synthesis telescopes.

\section{DISCUSSION \& CONCLUSIONS}

In the previous section, we have measured the structure and kinematics of six edge-on galaxies. For each of these galaxies, we presented a fit that assumed an optically thin medium, 

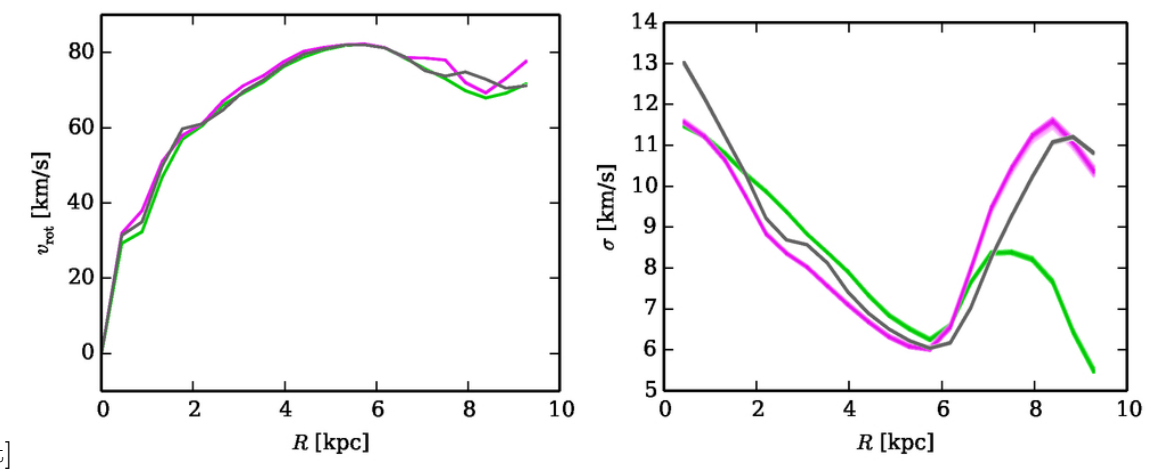

Figure 17. Fits to the rotation curve and velocity dispersion as function of height. Measurement in ESO 274-G001. The grey curve is the fit to the total galaxy, the cyan is the fit to the central plane of the galaxy and the green curve is the fit to the data that excludes the central plane.

as well as fit of a self-absorption medium with a spin temperature of $100 \mathrm{~K}$. As already predicted in Section 3.4 not accounting for self-absorption leads to very different results than the self-absorption models give. The most striking of this is the face-on column density, which is often measured $25 \%$ lower in the inner parts of the optically thin models. Other problems that occur when assuming an optically thin medium are an overestimation of the thickness of the HI disc and a completely wrong velocity dispersion. In our test models (Section 3.4), the velocity dispersion tends to be too high in the inner parts and too low in the outer part, when fitting the optically thin results to a self-absorption model, although this exact behaviour might not be universal.

The implication of this work is that the HI mass, thickness and velocity dispersion of galaxies are incorrect in the literature.

Comparing the optically thin results to those published in the literature, we find that our optically thin result match well. Only the velocity dispersion of the optically thin model appear very different compared to the work by O'Brien et al. (2010a). This difference is not fully understood, but is most likely due to the $z$-integrated PV diagram fitting by O'Brien et al. (2010a). Self-absorption has the effect of flattening Gaussian profile of the $\mathrm{HI}$ at a particular radius. As the data is integrated with height, it will lead to a different observed slope at the outer edges, than would appear in a normal Gaussian profile.

In Table 1, we show the list of all HI masses as measured using our outer envelope strategy. On average we find that $27 \pm 6 \%$ of the HI mass is hidden by self-absorption. In Section 7 of Paper II, we predicted that self-absorption would hide $30 \%$ of the mass if galaxy NGC 2403 had been viewed edge-on. These results are thus compatible. However, we stress that our analysis is based on a couple of important assumption. The first is the constant spin temperature of $100 \mathrm{~K}$. While this appears to work well for the data, its value is chosen only for convenience. In reality, the spin temperature is not constant, but is dependent on location inside the galaxy and the state of the HI gas. Assuming a lower average spin temperature would have resulted in a much higher total HI mass.

The second assumption is our treatment of the gas as a uniform medium. As we already discussed in Section 2.3 of Paper II, this is not realistic. In our Galaxy, many lines of sight are known to be optically thick within a couple of hundred parsec, with the highest concentrations of HI forming into distinct cloud structures (Taylor et al. 2003. Allen et al. 2012). The simulation of the Galactic plane by Douglas et al. (2010) shows that the opacity $\tau_{\nu}$ can go well above 25. Due to beam smearing and the large distances, we cannot resolve those cloud structures in our galaxies and as such, a uniform medium is justified. We stress however that because of the cloud structure of the HI , it could well hiding even more HI in the densest parts of these clouds. An analysis of the cloud structure in the nearby face-on galaxy M 31 by Braun (2012), showed that $34 \%$ of the HI was already hidden. The edge-on galaxies analyzed here could thus hide far more HI than was measured here.

With these assumptions in mind, we conclude that our new fitting strategy, along with the presented self-absorption models, are a more accurate representation of the neutral hydrogen content of edge-on galaxies, than the opticallythin decomposition strategies presented here and by other authors.

\section{ACKNOWLEDGMENTS}

SPCP is grateful to the Space Telescope Science Institute, Baltimore, USA, the Research School for Astronomy and Astrophysics, Australian National University, Canberra, Australia, and the Instituto de Astrofisica de Canarias, La Laguna, Tenerife, Spain, for hospitality and support during short and extended working visits in the course of his $\mathrm{PhD}$ thesis research. He thanks Roelof de Jong and Ron Allen for help and support during an earlier period as visiting student at Johns Hopkins University and the Physics and Astronomy Department, Krieger School of Arts and Sciences for this appointment.

PCK thanks the directors of these same institutions and his local hosts Ron Allen, Ken Freeman and Johan Knapen for hospitality and support during many work visits over the years, of which most were directly or indirectly related to the research presented in this series op papers.

Work visits by SPCP and PCK have been supported by an annual grant from the Faculty of Mathematics and Natural Sciences of the University of Groningen to PCK accompanying of his distinguished Jacobus C. Kapteyn professorhip and by the Leids Kerkhoven-Bosscha Fonds. PCK's 


\begin{tabular}{lrr} 
Name & Optically thin mass $\left[\mathrm{M}_{\odot}\right]$ & Self-absorbing mass $\left[\mathrm{M}_{\odot}\right]$ \\
\hline \hline IC 5052 & $7.4 \pm 0.7 \times 10^{8}$ & $9.5 \pm 0.9 \times 10^{8}$ \\
IC 5249 & $4.8 \pm 0.2 \times 10^{9}$ & $7.8 \pm 0.8 \times 10^{9}$ \\
ESO 115-G021 & $5.6 \pm 0.2 \times 10^{9}$ & $7.2 \pm 0.1 \times 10^{9}$ \\
ESO 138-G014 & $3.4 \pm 0.1 \times 10^{9}$ & $4.6 \pm 0.2 \times 10^{9}$ \\
ESO 274-G001 & $3.1 \pm 0.02 \times 10^{8}$ & $4.1 \pm 0.1 \times 10^{8}$ \\
UGC 7321 & $8.9 \pm 0.4 \times 10^{8}$ & $1.17 \pm 0.02 \times 10^{9}$ \\
\hline \hline
\end{tabular}

Table 1. The total HI mass of each galaxy as measured with our outer envelope strategy assuming either an optically thin or a self-absorbing mass.

work visits were also supported by an annual grant from the Area of Exact Sciences of the Netherlands Organisation for Scientific Research (NWO) in compensation for his membership of its Board.

\section{REFERENCES}

Abe F., Bond I. A., Carter B. S. a. e., 1999, AJ, 118, 261 Allen R. J., Ivette Rodríguez M., Black J. H., Booth R. S., 2012, AJ, 143, 97

Braun R., 2012, ApJ, 749, 87

Celnik W., Rohlfs K., Braunsfurth E., 1979, A\&A, 76, 24

Douglas K. A., Acreman D. M., Dobbs C. L., Brunt C. M., 2010, MNRAS, 407, 405

García-Ruiz I., Sancisi R., Kuijken K., 2002, A\&A, 394, 769

Gentile G., Fraternali F., Klein U., Salucci P., 2003, A\&A, 405, 969

Kregel M., van der Kruit P. C., 2004, MNRAS, 355, 143

Kregel M., van der Kruit P. C., de Blok W. J. G., 2004, MNRAS, 352, 768

Matthews L. D., Wood K., 2003, ApJ, 593, 721

O'Brien J. C., Freeman K. C., van der Kruit P. C., 2010a, A\&A, 515, A62

O'Brien J. C., Freeman K. C., van der Kruit P. C., 2010b, A\&A, 515, A61

O'Brien J. C., Freeman K. C., van der Kruit P. C., 2010c, A\&A, 515, A63

O’Brien J. C., Freeman K. C., van der Kruit P. C., Bosma A., 2010, A\&A, 515, A60

Olling R. P., 1996a, AJ, 112, 457

Olling R. P., 1996b, AJ, 112, 481

Sancisi R., Allen R. J., 1979, A\&A, 74, 73

Takamiya T., Sofue Y., 2002, ApJl, 576, L15

Taylor A. R., Gibson S. J., Peracaula M., et al., 2003, AJ, 125,3145

Uson J. M., Matthews L. D., 2003, AJ, 125, 2455

van der Kruit P. C., 1981, A\&A, 99, 298

van der Kruit P. C., Freeman K. C., 2011, ARA\&A, 49, 301

van der Kruit P. C., Jiménez-Vicente J., Kregel M., Freeman K. C., 2001, A\&A, 379, 374

This paper has been typeset from a $\mathrm{T}_{\mathrm{E}} \mathrm{X} / \mathrm{LT}_{\mathrm{E}} \mathrm{X}$ file prepared by the author. 